\title{
Analysis of X-Band Link Performance Degradation Caused by Adjacent Satellite
}

\author{
Durk-Jong Park ${ }^{1 \dagger}$, Sang-Il Ahn ${ }^{1}$, Yong-Sik Chun ${ }^{1}$, and Eun-Kyou Kim ${ }^{2}$ \\ ${ }^{1}$ Ground System Development Team, Korea Aerospace Research Institute, Daejeon 305-333, Korea \\ ${ }^{2}$ Satellite Operation Division, Korea Aerospace Research Institute, Daejeon 305-333, Korea
}

\begin{abstract}
As more satellites are designed to downlink their observed image data through the X-band frequency band, it is inevitable that the occupied bandwidth of a target satellite will overlap with that of other X-band downlink satellites. For sun-synchronized low earth orbit satellites, in particular, it can be expected that two or more satellites be placed within the looking angle of a ground station antenna at the same time. Due to the overlapping in the frequency band, signals transmitted from the adjacent satellites act as interferers, leading to degraded link performance between target satellite and ground station. In this paper, link analysis was initiated by modeling the radiation pattern of ground station antenna through a validated Jet Propulsion Laboratory peak envelope model. From the relative antenna gain depending on the offset angle from center axis of maximum antenna directivity, the ratio of received interference signal level to the target signal level was calculated. As a result, it was found that the degradation increased when the offset angle was within the first null point of radiation pattern. For a $7.3 \mathrm{~m}$ antenna, serious link degradation began at an offset angle of 0.4 degrees. From this analysis, the link performance of the coming satellite passes can be recognized, which is helpful to establish an operating procedure that will prevent the ground station from receiving corrupted image data in the event of a degraded link.
\end{abstract}

Keywords: interference, X-band, adjacent satellite

\section{INTRODUCTION}

Earth observation satellites such as TERRA and AQUA satellites are supposed to transmit their observed information or imaging data through the X-Band frequency band $(8,025 \sim 8,400 \mathrm{MHz})$, which has already been allocated by the International Telecommunication Union Radiocommunication sector (ITU-R). On the other hand, the need to transmit high-resolution image data has recently emerged. For this reason, band-efficiency schemes such as dual polarization transmission have been applied (Maral et al. 1993). However, the allocated frequency band has been fully occupied due to the use of earth observation satellites with high-resolution sensors. For example, in the case of the Korea Multi-Purpose SATellite-2 (KOMPSAT-2), the center frequency and bandwidth of X-band downlink signal are $8,205 \mathrm{MHz}$ and $320 \mathrm{MHz}$, respectively. Meanwhile, the center frequency and bandwidth of the AQUA satellite are 8,160 MHz and $15 \mathrm{MHz}$. Therefore, $\mathrm{X}$-band downlink signal of AQUA is fully located at the in-band of KOMPSAT-2. Since the local time ascending node (LTAN) of each is different, there is no possibility of seeing dual-contact in KARI site, Daejeon. However, in the case of KOMPSAT-3, the considered LTAN is around 13 hours 30 minutes, which causes dual-contact with the AQUA satellite in a single ground station. Furthermore, the AQUA satellite is designed to keep broadcasting its Xband observation data to any ground station in the alongtracking path. For this reason, AQUA satellite can easily be expected to act as an interferer to the ground station that is supposed to be receiving KOMPSAT-3 image data.

Fig. 1 shows the analysis procedure applied in this pa- (c) This is an Open Access article distributed under the terms of the Creative Commons Attribution Non-Commercial License (http://creativecommons.org/licenses/by-nc/3.0/) which permits unrestricted non-commercial use, distribution, and reproduction in any medium, provided the original work is properly cited.
Received Sep 26, 2011 Revised Oct 24,2011 Accepted Nov 02, 2011 ${ }^{\dagger}$ Corresponding Author

E-mail: parkdj@kari.re.kr

Tel: +82-42-860-2721 Fax: +82-42-870-3919 
per. For the calculation of the interference signal level, relative antenna gain vs. offset angle needs to be recognized in advance. Here, offset angle is the angle difference between target satellite and interference satellite. Since it is baseline that ground station antenna is tracking to the target satellite with its maximum gain, the interference signal level received at the ground station antenna is determined depending on the relative antenna gain of the ground station antenna. The relative antenna gain can be obtained from the modeling of an antenna radiation pattern using the Jet Propulsion Laboratory (JPL) peak envelope model, which has already been validated in largescale antenna applications of which the diameter versus wavelength is over 100 (Jamnejad \& Peng 2006).

From the radiation pattern of the ground station modeled by the JPL peak envelope model, the signal level of the adjacent satellite received at the ground station is calculated using the relative antenna gain at any offset angle. Once the signal level of the adjacent satellite is obtained, it needs to be reflected into link analysis through key link equations. These key equations were validated by simulating a dual-contact scenario. After conducting validation on the key link equations, link analyses were conducted in the dual-contact case. Here, AQUA satellite was assumed to be the adjacent satellite. Through link analysis, the degradation caused by adjacent satellites was calculated according to antenna size, elevation angle and offset angle.

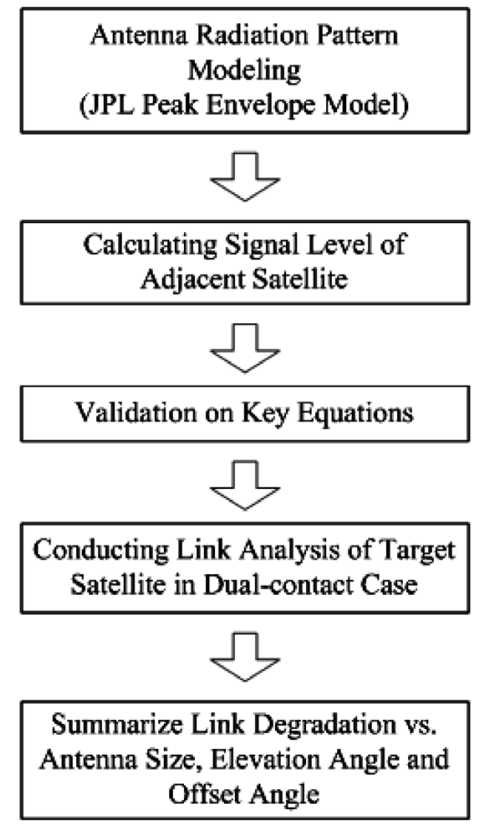

Fig. 1. Analysis procedure for dual-contact scenario.

\section{ANTENNA OFFSET ANGLE}

Fig. 2 illustrates the offset angle between target satellite and interference satellite. The meaning of the offset angle is the angle difference between the target satellite and the adjacent satellite. Normally, ground station antenna is supposed to perform tracking on the target satellite with its maximum antenna gain. Therefore, for the adjacent satellite, signal level at the antenna output of ground station is determined through the relative antenna gain corresponding to the offset angle of the ground station antenna.

To calculate the offset angle, it is necessary to recognize azimuth and elevation angle of the ground station antenna in the direction of the target satellite and the adjacent satellite, respectively. The angle information can be obtained from orbit analysis based on the geo-location of ground station and two-line element file of each satellite. As a result of orbit analysis, antenna looking angle for target satellite and adjacent satellite can be expressed as azimuth angle $\left(\phi, \phi^{\prime}\right)$ and elevation angle $\left(\theta, \theta^{\prime}\right)$ in spherical coordination. After transforming the angle elements as in rectangular coordination, Offset angle can be calculated in the form:

$$
\operatorname{Offset} \text { Angle }(\gamma)=\arccos \left(\frac{\vec{a} \cdot \vec{b}}{|\vec{a}| \mid \vec{b}}\right)
$$

here, $\vec{a}$ is a vector when a ground station antenna is looking for the target satellite, and $\vec{b}$ is for the adjacent satellite.

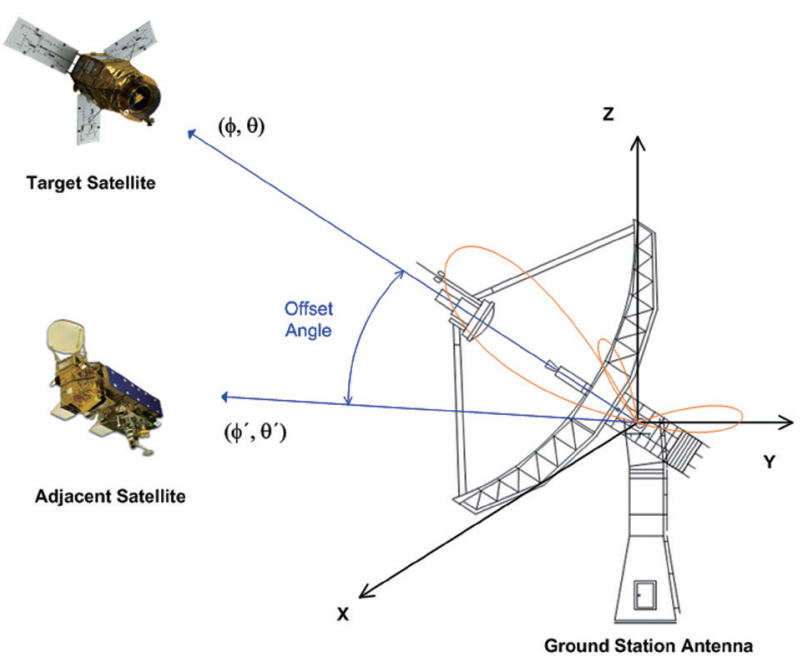

Fig. 2. Offset angle between target satellite and adjacent satellite. 


\section{ANTENNA RADIATION PATTERN}

Table 1 shows the offset angle corresponding to half power beam width (HPBW), 1st null point and 1st side lobe at 8,205 MHz, which are obtained from the modeled $13 \mathrm{~m}, 11 \mathrm{~m}$ and $7.3 \mathrm{~m}$ antennas. Three different antennas have been used for KOMPSAT-2 satellite operation at Daejeon and Seoul. Indeed, those parabolic antennas will be applied for receiving X-Band image data from KOMPSAT-3 satellite operation.

Compared with the measured HPBW, 1st null point, 1st side lobe of $7.3 \mathrm{~m}$ antenna manufactured by ViaSat, it was found that the JPL peak envelope model was accurate, with 0.125 degrees of difference. The radiation pattern of $13 \mathrm{~m}$ antenna was so sharp that the 1 st null point was 0.23 degrees, while that of the $7.3 \mathrm{~m}$ antenna was 0.4 degrees.

\section{KEY EQUATIONS}

The overall Eb/No (ratio of bit energy to noise power density) in a dual-polarized communication link, $\left(E_{b} / N_{0}\right)_{\text {overall }}$, is expressed in the following (Vasseur 2000).

$$
\left(E_{b} / N_{0}\right)_{\text {overall }}^{-1}=\left(E_{b} / N_{0}\right)_{s}^{-1}+\left(E_{b} / N_{0}\right)^{-1}
$$

Here, $\left(E_{b} / N_{0}\right)_{s}$ is Eb/No in a single-polarized communication link while $\left(E_{b} / I_{0}\right)$ is the ratio of bit energy to the power density of interference signal. In decibel units, $\left(E_{b} / I_{0}\right)$ is expressed as follows (Vasseur 2000):

Table 1. Offset angle for angle for HPBW, 1st null point, and 1st side lobe of $13 \mathrm{~m}, 11 \mathrm{~m}$ and $7.3 \mathrm{~m}$ antennas at $8,205 \mathrm{MHz}$

\begin{tabular}{lccc}
\hline \multirow{2}{*}{ Items (degrees) } & \multicolumn{3}{c}{ Antenna size } \\
\cline { 2 - 4 } & $\mathbf{7 . 3 \mathbf { ~ m }}$ & $\mathbf{1 1 ~ \mathbf { ~ m }}$ & $\mathbf{1 3 ~ \mathbf { ~ }}$ \\
\hline HPBW & 0.17 & 0.11 & 0.10 \\
1st null point & 0.40 & 0.27 & 0.23 \\
1st side lobe & 0.70 & 0.47 & 0.40 \\
\hline
\end{tabular}

HPBW: half power beam width.

Table 2. Initial setup for validation test.

\begin{tabular}{lc}
\hline \multicolumn{1}{c}{ Parameters } & Values \\
\hline Signal level at test modulator \#1 & $-23.5 \mathrm{dBm}$ \\
Noise level at test modulator \#2 & $-119.5 \mathrm{dBm} / \mathrm{Hz}$ \\
Measured BER & $3.30 \times 10^{-6}$ \\
Reference Eb/No & $10.34 \mathrm{~dB}$ \\
Measured Eb/No & $10.94 \mathrm{~dB}$ \\
Implementation loss & $0.6 \mathrm{~dB}$ \\
\hline
\end{tabular}

BER: bit error rate.

$$
\left(E_{b} / I_{0}\right)=C / I+10 \log _{10}(B / R)
$$

Here, $C$ is un-modulated signal level and $I$ is interference signal level. In addition, $B$ and $R$ is occupied bandwidth and bit rate, respectively. In OQPSK modulation, the ratio of bandwidth to bit rate is same. Therefore, carrier to interference signal ratio can be expressed as follows:

$$
(C / I)^{-1}=\left(X P D_{\text {total }}\right)^{-1}+\left(C / I_{\text {others }}\right)^{-1}
$$

Here, $X P D_{\text {total }}$ refers to the overall cross polarization discrimination (XPD), while $C / I_{\text {others }}$ is the ratio of signal level received from target satellite to signal level received from adjacent satellite.

Fig. 3 shows the test configuration for the validation of the key equations. The aim of conducting this validation test is to see if degradation on $\mathrm{Eb} / \mathrm{No}$ is similar to the expected value based on key equations. Firstly, reference $\mathrm{Eb} / \mathrm{No},(\mathrm{Eb} / \mathrm{No})_{r e f}$, is measured when only test modulator \#1 is generating an interesting signal, like the target satellite. Table 2 shows the initial setup including reference $\mathrm{Eb} /$ No for validation test.

Test modulation \#1 and \#2 are working to generate test signals modulated through OQPSK with a data rate of 320 Mbps, which will be applied for one of two X-band downlink channels of the KOMPSAT-3 satellite. Test modulator \#2 was working to generate a test signal corresponding to the XPD and signal of the adjacent satellite. Two signals generated by test modulator \#1 and test modulator \#2 were supposed to be combined at power combiner. Due

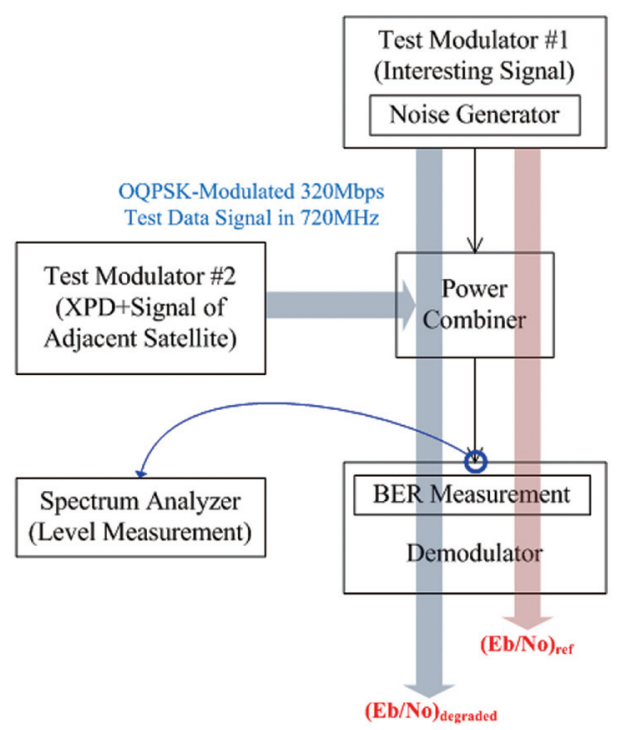

Fig. 3. Test configuration for validation on key equations. XPD: cross polarization discrimination, BER: bit error rate. 
Table 3. Result of validation test.

\begin{tabular}{ccccccc}
\hline $\begin{array}{c}\text { Signal level at } \\
\text { test modulator \#2 } \\
(\mathbf{d B m})\end{array}$ & $\begin{array}{c}\text { XPD and signal } \\
\text { level of adjacent } \\
\text { satellite }(\mathbf{d B})\end{array}$ & $\begin{array}{c}\text { Measured BER } \\
\left(\times \mathbf{1 0}^{-6}\right)\end{array}$ & $\begin{array}{c}(\mathbf{E b} / \mathbf{N o})_{\text {degraded }} \\
(\mathbf{d B})\end{array}$ & $\begin{array}{c}\text { Measured } \\
\text { degradation } \\
(\mathbf{d B})\end{array}$ & $\begin{array}{c}\text { Calculated } \\
\text { degradation in link } \\
\text { analysis (dB) }\end{array}$ & $\begin{array}{c}\text { Difference } \\
(\mathbf{d B})\end{array}$ \\
\hline-50.0 & 26.5 & 5.4 & 10.2 & 0.2 & 0.12 & 0.15 \\
-49.0 & 25.5 & 6.0 & 10.1 & 0.2 & 0.19 & 0.1 \\
-48.0 & 24.5 & 7.0 & 10.0 & 0.3 & 0.23 & 0.1 \\
-47.0 & 23.5 & 8.2 & 10.0 & 0.4 & 0.28 & 0.1 \\
-46.2 & 22.7 & 9.5 & 9.9 & 0.4 & 0.35 & 0.2 \\
-45.2 & 21.7 & 12.0 & 9.8 & 0.5 & 0.44 & 0.2 \\
-44.2 & 20.7 & 15.0 & 9.7 & 0.6 & 0.54 & 0.2 \\
-43.2 & 19.7 & 19.0 & 9.6 & 0.7 & 0.66 & 0.2 \\
-42.3 & 18.8 & 24.0 & 9.5 & 0.8 & 0.81 & 0.2 \\
-41.3 & 17.8 & 32.0 & 9.4 & 1.0 & 1.00 & 0.2 \\
-40.3 & 16.8 & 48.0 & 9.2 & 1.2 & 1.18 & 0.2 \\
-39.5 & 16.0 & 68.0 & 9.0 & 1.3 & & 0.2 \\
\hline
\end{tabular}

XPD: cross polarization discrimination.

Table 4. Key link parameters between target satellite and ground station antenna.

\begin{tabular}{lccc}
\hline \multicolumn{1}{c}{ Parameters } & \multicolumn{3}{c}{ Values } \\
\hline Frequency $(\mathrm{MHz})$ & \multicolumn{3}{c}{8205} \\
S/C height $(\mathrm{km})$ & \multicolumn{3}{c}{45.13 .76} \\
S/C EIRP $(\mathrm{dBm})$ & \multicolumn{3}{c}{0.8} \\
S/C antenna axial ratio (dB) & \multicolumn{3}{c}{ 26.5 } \\
S/C antenna XPD (dB) & ITU model applied (ITU-R 2001) \\
Atmopheric loss & $13 \mathrm{~m}$ & $11 \mathrm{~m}$ & $7.3 \mathrm{~m}$ \\
Size of ground station antenna & 0.27 & 0.27 & 0.27 \\
Pointing koss (dB) & 26.5 & 26.5 & 26.5 \\
Ground antenna XPD (dB) & 36.5 & 35.05 & 31.49 \\
G/T (dB/K) &
\end{tabular}

EIRP: equivalent isotropically radiated power, XPD: cross polarization discrimination, ITU-R: International Telecommunication Union Radiocommunication.

Table 5. Major link parameters of AQUA satellite.

\begin{tabular}{lc}
\hline \multicolumn{1}{c}{ Parameters } & Values \\
\hline Frequency $(\mathrm{MHz})$ & 8,160 \\
S/C height $(\mathrm{km})$ & 705 \\
S/C EIRP $(\mathrm{dBm})$ & 32.22 to 46.52 \\
S/C antenna axial ratio $(\mathrm{dB})$ & 3.2 \\
\hline EIRP: equivalent isotropically radiated power.
\end{tabular}

to the combined signal, link degradation was reflected on the measured $\mathrm{Eb} / \mathrm{No},(\mathrm{Eb} / \mathrm{No})_{\text {degraded }}$. Consequently, degradation can be measured as the difference between reference $\mathrm{Eb} / \mathrm{No}$ and degraded $\mathrm{Eb} /$ No. Table 3 shows the result of the validation test. The calculated degradation was obtained using Eqs. (2-4), and it was found that the difference between measured degradation and calculated degradation was maintained within $0.2 \mathrm{~dB}$. Since the measurement accuracy of spectrum analyzer was $0.5 \mathrm{~dB}$, it could be determined that accuracy within $0.2 \mathrm{~dB}$ was acceptable for link analysis.

\section{LINK ANALYSIS RESULTS}

As an example for the link analysis, Table 4 shows link parameters between the target satellite and ground station antenna.

Table 5 shows the relevant link parameters of AQUA satellite in A-Train group, which was assumed as an adjacent satellite in this paper. A-Train is the afternoon constellation consisting of several earth observation satellites belonging to National Aeronautics and Space Administration and Centre National d'Etudes Spatiales (Goddard Space Flight Center 2003). Regarding the spacecraft equivalent isotropically radiated power, on-board antenna gain of AQUA satellite was designed to differ depending on the angle from antenna center axis (Goddard Space Flight Center 2002). The highest antenna gain is placed at an off-nadir angle of about 64 degrees, while the lowest one is placed at an off-nadir angle of 17 degrees.

Fig. 4 shows, through the link analysis, that degradation was dependent on the angle from peak of main beam in $7.3 \mathrm{~m}$ antenna. Link degradation significantly increases from an offset angle of 0.4 degrees, which corresponds to the 1st null of a $7.3 \mathrm{~m}$ antenna radiation pattern. Since the relative antenna gain was increasing from the 1st null point to the offset angle of zero degrees, the received interference signal was also increasing. On the other hand, it was shown that degradation in an elevation angle of 60 degrees was lower than even the case of 90 degrees elevation angle. This was due to a characteristic of the AQUA on-board antenna in which the lowest relative antenna 
gain was located at 17 degrees from on-board antenna center axis.

Fig. 5 shows the link margin and degradation versus

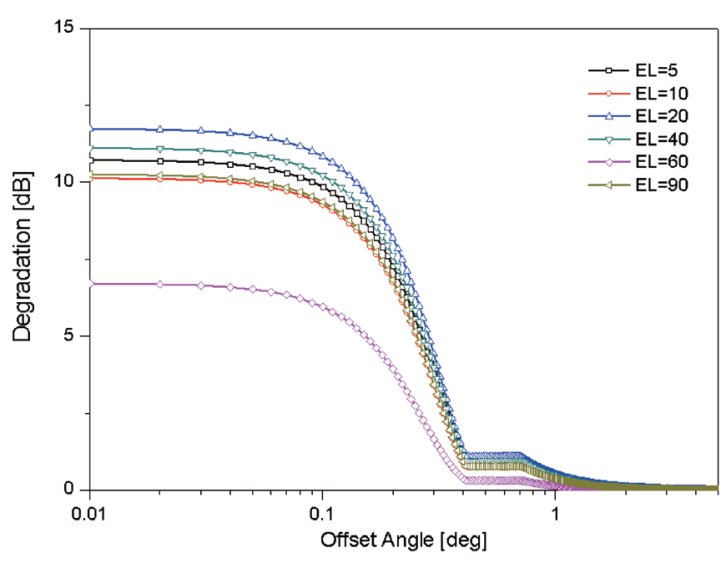

Fig. 4. Degradation versus offset angle.

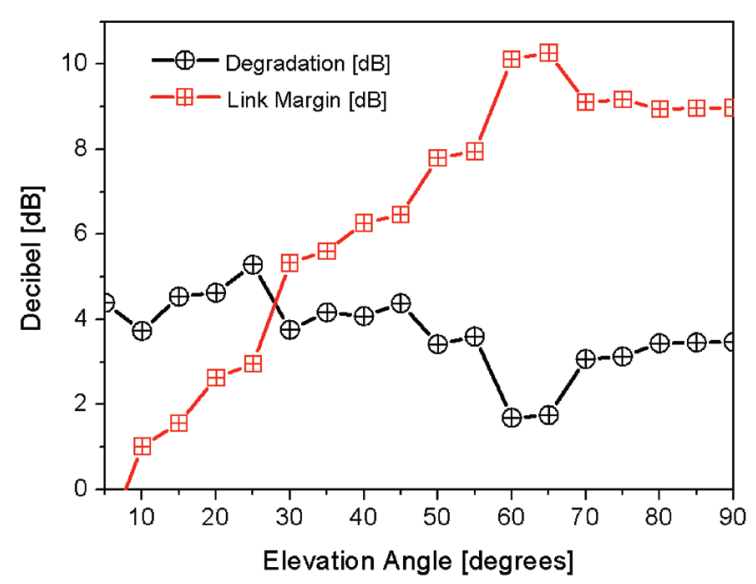

Fig. 5. Degradation and link margin versus elevation angle.

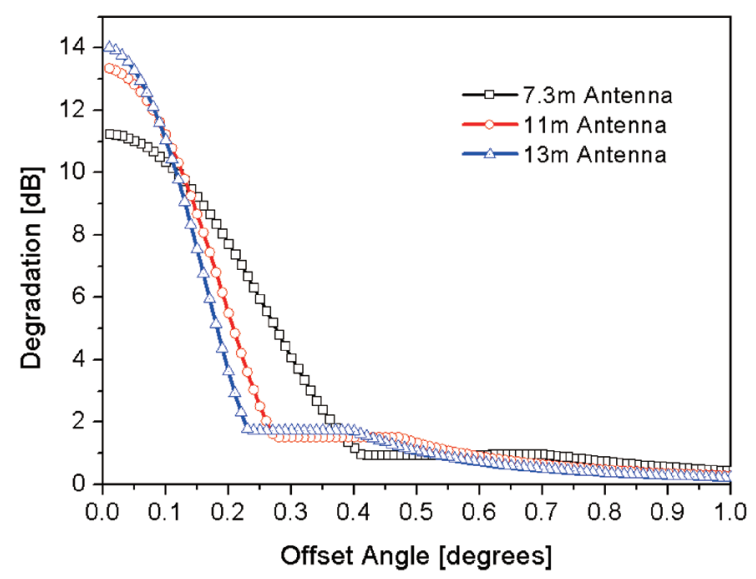

Fig. 6. Degradation versus offset angle. elevation angle, assuming that the offset angle was 0.3 degrees. According to the characteristics of the on-board antenna of the AQUA satellite, the degradation caused by the AQUA satellite was the lowest near an elevation angle of 60 degrees. On the other hand, link margin was continuously increasing, which was dependent on the elevation angle. The reason for increasing link margin was that distance loss between satellite and ground station was lower when elevation angle was 90 degrees.

Fig. 6 shows the degradation depending on the antenna size, assuming that elevation angle was 40 degrees and AQUA satellite was working as an adjacent satellite. It was shown that the offset angle where the degradation was dramatically increasing differed depending on the size of the antenna. According to the 1st null point of each antenna, the relevant offset angle was around 0.4 degrees in a $7.3 \mathrm{~m}$ antenna, 0.27 degrees in an $11 \mathrm{~m}$ antenna, and 0.23 degrees in a $13 \mathrm{~m}$ antenna.

\section{CONCLUSIONS}

In this paper, link degradation of target satellite was analyzed assuming that an adjacent satellite was also placed in the looking angle of the ground station antenna. Since the X-band for earth observation has been nearly occupied these days, degradation is expected due to the frequency overlap in the in-band of target satellite. For the analysis of the degradation, JPL peak envelope model was applied to model the radiation pattern of the ground station antenna. From the antenna radiation pattern, relative antenna gain was calculated depending on the offset angle. Link equations were validated through bit error rate measurement. As a result of link analysis, assuming that AQUA satellite was an adjacent satellite, it was shown that link degradation was significantly increased from the offset angle related with the 1st null point of ground station antenna. In terms of satellite operation, an analysis of the degradation should be conducted when an adjacent satellite is expected to be in the antenna looking angle. If huge degradation is recognized, then mission planning should be modified to allow the regional-diversity and timing-diversity ground station to receive image data.

\section{REFERENCES}

Goddard Space Flight Center, Interface description document for EOS AQUA X-band direct broadcast [GSFC 
422-11-19-11] (Goddard Space Flight Center, Greenbelt, 2002).

Goddard Space Flight Center, Formation flying: the afternoon "A-Train" satellite constellation [FS-2003-1-053GSFC] (Goddard Space Flight Center, Greenbelt, 2003).

ITU Radiocommunication (ITU-R), Propagation data and prediction methods for the design of earth-space telecommunication systems [ITU-R P.618-7] (2001).

Jamnejad V, Peng T, Mathematical gain models of large-aperture Earth station antennas for Space Research Service, in IEEE Aerospace Conference, Big Sky, MT, 2006. http:// dx.doi.org/10.1109/AERO.2006.1655811

Maral G, Bousquet M, Nelson JCC, Satellite communication systems: systems, techniques and technologies, 4 th ed. (John Wiley and sons, Chichester, 2003), 198.

Vasseur H, Degradation of availability performance in dual-polarized satellite communications systems, IEEE Trans Commun, 48, 465-472 (2000). http://dx.doi.org/ 10.1109/26.837049 